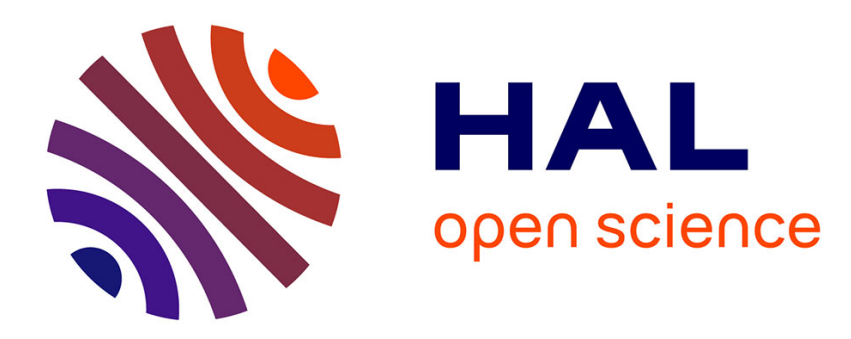

\title{
CURRENT-DRIVEN TEXTURAL TRANSITION OF 3He-A
}

Chia-Ren Hu, T. Ham

\section{To cite this version:}

Chia-Ren Hu, T. Ham. CURRENT-DRIVEN TEXTURAL TRANSITION OF 3He-A. Journal de Physique Colloques, 1978, 39 (C6), pp.C6-55-C6-56. 10.1051/jphyscol:1978626 . jpa-00217695

\section{HAL Id: jpa-00217695 https://hal.science/jpa-00217695}

Submitted on 1 Jan 1978

HAL is a multi-disciplinary open access archive for the deposit and dissemination of scientific research documents, whether they are published or not. The documents may come from teaching and research institutions in France or abroad, or from public or private research centers.
L'archive ouverte pluridisciplinaire HAL, est destinée au dépôt et à la diffusion de documents scientifiques de niveau recherche, publiés ou non, émanant des établissements d'enseignement et de recherche français ou étrangers, des laboratoires publics ou privés. 
CURRENT-DRIVEN TEXTURAL TRANSITION OF ${ }^{3} \mathrm{He}-\mathrm{A}^{+}$

Chia-Ren Hu and T.E. Ham

Department of Physies, Texas A\&M University, College Station, Texas 77843 USA

Résumé.- En utilisant une méthode variationnelle, nous étudions la transition hydrodynamique de la texture de ${ }^{3} \mathrm{He}-\mathrm{A}$ dans une géométrie planaire pour des températures proches de $\mathrm{T}_{\mathrm{C}}$ et une épaisseur de l'échantillon $\gg 10^{-3} \mathrm{~cm}$, sans nous limiter à des textures bidimensionnelles. On trouve que la transition est du deuxième ordre mais très proche d'être du premier lorsqu'on varie le courant.

Abstract.- Using a variational approach, we study the current-driven textural transition of ${ }^{3} \mathrm{He}-\mathrm{A}$ in a slab geometry, for temperatures near the critical, and slab width $\gg 10^{-3} \mathrm{~cm}$, without restricting ourselves to only planar textures. The transition is found to be 2 nd order, but very close to being first order, as current is varied.

Recently, Fetter has shown that the uniform texture of ${ }^{3} \mathrm{He}-\mathrm{A}$ in a slab has a hydrodynamic instability at a critical wave number $Q_{c} / 1 /$. His assumption of a planar texture for $Q>Q_{c}$, however, has since been realized to be invalid because the exact equilibrium conditions indicate that a flow can exert a torque to rotate the orbital axis $\hat{\ell}$ out of a planar configuration $/ 2 /$. In this paper, therefore, we reexamine this instability without the restriction to planar textures, and also attempt to establish the order of the transition that occurs at $\mathbf{Q}_{\mathrm{c}}$.

The orbital part of the order parameter for ${ }^{3} \mathrm{He}-\mathrm{A}$ is $\Delta_{0}(\hat{\mathrm{m}}+\hat{\mathrm{in}})$ where $\hat{\mathrm{m}}, \hat{\mathrm{n}}(\underline{\mathrm{m}})$ and $\hat{\ell}=\hat{\mathrm{m}} \times \hat{\mathrm{n}}$ are a triad of three unit vectors $/ 2 /$. Here it is convenient to let $/ 3 /$ :

$$
\begin{aligned}
\hat{\mathrm{n}}+\mathrm{i} \hat{\mathrm{n}} & =e^{\mathrm{i} Q \mathrm{y}}\left[\mathrm{A}_{0} \hat{\mathrm{x}}+A_{+}(\hat{\mathrm{y}}+\mathrm{i} \hat{z}) / \sqrt{2}+A_{-}(\hat{y}-i \hat{z}) / \sqrt{2}\right. \\
A_{0} & =\sin \chi \exp \left(i S_{1}\right) \\
A_{ \pm} & =(1 / \sqrt{2})(\cos \mathrm{X} \pm 1) \exp \left[\hat{i}\left(S_{1} \pm S_{2}\right)\right]
\end{aligned}
$$

where the slab boundaries are assumed to be located at $x=0$ and $w$, and the flow is taken to be along $y$. This representation gives : $\ell_{x}=\cos x$, $\ell_{y}=-\sin \chi \cos s_{2}, l_{z}=\sin x \sin s_{2}$. The gradient energy density near $T_{c}$, after assuming that $s_{1,2}$ and $x$ depend only on $x$, reduces to :

$$
\begin{aligned}
& f_{G}=\Delta_{0}^{-2}\left\{\left(\Gamma_{13} \sin ^{2} \chi+2 \Gamma_{2}\right) S_{1 x}{ }^{2}\right. \\
& +\left[\Gamma_{13}\left(\sin ^{2} S_{2}+\cos ^{2} x \cos ^{2} S_{2}\right)+2 \Gamma_{2}\right] Q^{2} \\
& +2 \Gamma_{13} Q \sin \chi \cos S_{2}\left(\cos x S_{1 x}+S_{2 x}\right) \\
& \left.-2 \Gamma_{1} Q(\sin x \operatorname{sins})_{2}\right)+4 \Gamma_{2} \cos x S_{1 x} S_{2 x} \\
& \left.+\left(\Gamma_{13}+3 \Gamma_{2}\right) x_{x}{ }^{2}+\left[\Gamma_{13} \sin ^{4} \chi+\Gamma_{2}\left(2+\sin ^{2} x\right)\right] s_{2 x}{ }^{2}\right]
\end{aligned}
$$

\footnotetext{
$\mp$ Supported by National Science Foundation under Grant No DMR76-81328
}

where the parameters $\Gamma_{13} \equiv \Gamma_{1}+\Gamma_{3}$ and $\Gamma_{2}$ have been defined in reference $/ 4 /$, and we have assumed dipole-locking of the spin axis $a$ with $\downarrow$, thereby restricting ourselves to $\mathrm{w}>>10^{-3} \mathrm{~cm}$. An expression for the current density along $\hat{y}$ may be obtained from $/ 1 /: j_{y}=(2 m / h)\left(\partial f_{G} / \partial Q\right)$. Minimizing $F_{f} \equiv f_{G} d^{3} x$ with respect to $s_{1}$ gives a current conservation equation and boundary condition (along $\hat{x}$ ) which may be integrated once to allow $S_{1 x}$ to be eliminated in terms of $Q, S_{2}$, and $x$. If the resulting $F_{G}\left[x, s_{Z}\right]$ is further minimized, one obtains two coupled non-linear differential equations which must be solved numerically. [The boundary condition being $x=s_{2 x}=0$ at $x=0$ and $w$, where $\mathrm{s}_{2 \mathrm{x}}=0$ is required to prevent unphysical divergences.] While this numerical solution is currently being attempted, and results will be presented, below we wish to discuss the results of a semi-quantitative study, based on a variational approach : Approximating the trigonometric functions of $x$ and $S_{2}$ in $F_{G}$ by their leading one-or two-term expansions, depending on an order analysis which ensures that a11 terms of $4^{\text {th }}$ order or lower, and some of the terms of $6^{\text {th }}$ order and higher, are included, we then minimize $\mathrm{F}_{\mathrm{G}}\left[\mathrm{X}, \mathrm{S}_{2}\right]$ using the trial functions $x=\alpha \sin (\pi x / w), s_{2}=\beta \cos (\pi x / w)$, treating $\alpha$ and $\beta$ as variational parameters. It is found that the instability is at the same $Q_{c}$ as found by Fetter $/ 1 /$ (i.e., $Q_{c}=\sqrt{5} \pi / 2 w$ after assuming $\Gamma_{1}=\Gamma_{2}=$ $\Gamma_{3}$ ) but the inhomogeneous texture at $Q>Q_{c}$ is distinctly non-planar. For $\left(Q-Q_{c}\right) \ll Q_{c}$ when $\alpha$ and $\beta$ are small, the analysis may be done analytically by a systematic perturbation approach. Introducing the normalized total gradient energy and current : 


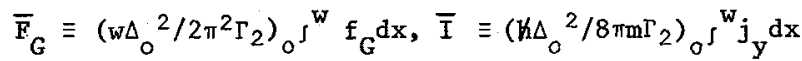
we find $: \beta=(3 / 5) q \alpha, \alpha^{2}=(8 / 11)\left(q^{2}-5 / 4\right)$ where $q$ $q \equiv Q w / \pi$, and $\bar{F}_{G}=2 q^{2}-(4 / 11)\left(q^{2}-5 / 4\right)^{2}$, $\bar{I}=2 q\left[1-(4 / 11)\left(q^{2}-5 / 4\right)\right]$. This should be compared with the values for $Q<Q_{c}: \alpha=\beta=0$, $\bar{F}_{G}=2 q^{2}$ and $\bar{I}=2 q$, corresponding to a uniform texture with $t=\hat{x}$, The slope $\partial \bar{I} / \partial q$ is seen to change from 2 for $Q<Q_{c}$, to $2 / 11$ for $Q>Q_{c}$. Since $\partial I / \partial q$ $>0$ above $Q_{c}$, the textural phase transition as predicted by the present approximate study is a secondorder transition with no hysteresis as current is varied, but the smallness of $2 / 11$ suggests that a more precise calculation using numerical methods might predict a first order transition at $Q_{c}$ (i.e., with $\partial I / \partial q<0$ just above $Q_{c}$ ). This interesting possibility is currently being explored. For larger values of $Q-Q_{c}$ we have minimized our $\bar{F}_{G}(\alpha, \beta)$ by numerical method. In figure 1 we have plotted the nor malized gradient energy $\bar{F}_{G} / 2 q^{2}$, and the normalized current $\bar{I}$, as function of $q$. The dotted lines indicate what would have been obtained if planar approximation were used (i.e. if $\beta=0$ is assumed).

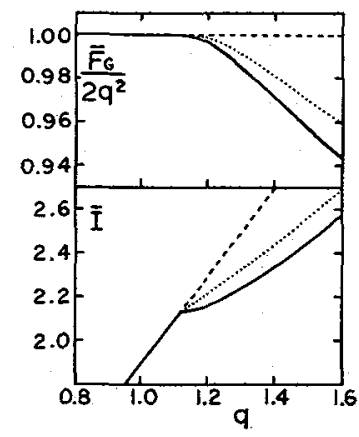

Fig. I : The normalized gradient energy $\overline{\mathrm{F}}_{\mathrm{G}} / 2 \mathrm{q}^{2}$, and total current $\bar{I}$, are plotted as functions of $q \equiv Q_{w} / \pi$ (solid lines). The dotted lines indicate the corresponding results if planar approximation were used.

In figure 2 we have plotted the values of $\alpha^{2}$ and $\beta^{2}$ which minimize $\bar{F}_{G}$, as function of $q$ (solid lines), as we11 as the value of $\alpha^{2}$ which minimizes $\left.\bar{F}_{G}\right|_{\beta=0}$ (the dotted line). We note also that for $q \gg 1$ (when our approximation breaks down) we expect $\bar{F}_{G} \rightarrow q^{2}$ and $\bar{I} \rightarrow q$ corresponding to a uniform texture with $\ell=\hat{y}$, except within surface regions of thickness $\sim Q^{-1} \ll w / 5 /$. In conclusion, we point out that the stability of the new inhomogeneous current-carrying state for $q^{2}>5 / 4$ has yet to be established, as is done in reference /5/ for $q \gg 1$, in order to confirm the textural transition at $Q_{c}$.

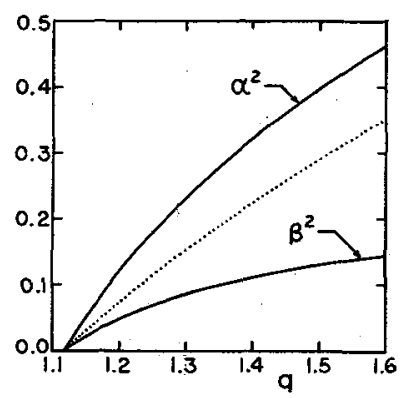

Fig. 2 : The squares of the variational parameters $\alpha$ and $\beta$ which minimize $\bar{F}_{G}$, are plotted as functions of $q$ (solid lines). The dotted line is the square of the value of $\alpha$ which minimizes $\left.\bar{F}_{G}\right|_{\beta=0}$

It is hoped that our current attempt for the exact solution of this problem by numerical method can be extended to include such a stability analysis.

\section{References}

/1/ Fetter,A.L., Phys. Rev. B14 (1976) 2801

/2/ See, for example Hall,H.E. and Hook, J.R., J. Phys. C10 (1977) L91; Hu, C.-R. ans Saslow, W.M., Phys. Rev. Lett. 38 (1977) 605 ; and Fetter, A.L., to be published in Phys. Rev. B 17 (1978) 1152 .

/3/ This is only a slight modification from a representation recently intoduced by : Tsuneto, T., Ohmi, T. and Fujita, T. to be published in Proceedings of International Symposium on Physics at Ultralow Temperatures (Hakone)

$. / 4 / \mathrm{Hu}$, C. - R., Ham, T.E. and Saslow, W., J. Low Temp. Phys. 32 (1978) 301, Eqs. (3) and (4).

15/ Bhattacharyya, P., Ho, T.-L. and Mermin, N.D., Phys. Rev. Lett. 39 (1977) 1290 